\title{
Contribution of visual velocity and displacement cues to human balancing of support surface tilt
}

\author{
Lorenz Assländer • Georg Hettich • Albert Gollhofer • \\ Thomas Mergner
}

\begin{abstract}
Vision helps humans in controlling bipedal stance, interacting mainly with vestibular and proprioceptive cues. This study investigates how postural compensation of support surface tilt is compromised by selectively reducing visual velocity cues by stroboscopic illumination of a stationary visual scene. Healthy adult subjects were presented with pseudorandom tilt sequences in the sagittal plane (tilt frequency range $0.017-2.2 \mathrm{~Hz}$; velocity amplitude spectrum constant up to a frequency of $0.6 \mathrm{~Hz}$, angular displacement amplitude spectrum increasing with decreasing frequencies). Center of mass (COM) sway responses were recorded for stroboscopic illuminations at $48,32,16$, 8 , and $4 \mathrm{~Hz}$, as well as under continuous illumination and with eyes closed. With strobe duration $(5 \mathrm{~ms})$ and mean luminance (1 lx) kept constant, visual acuity and perceived brightness remained constant and the visual scene was perceived as stationary. Yet, tilt-evoked COM excursions increased with decreasing strobe frequency in a graded way, with largest effects occurring at tilt frequencies where large tilt velocities coincided with small displacements. In addition, COM excursions were reduced at the lowest strobe frequency compared to eyes closed, with the largest effect occurring at tilt frequencies where tilt displacements were large. We conclude that two mechanisms exist, a velocity mechanism that deals with tilt compensation and is foremost affected by the stroboscopic illumination and a displacement mechanism. This compares favorably
\end{abstract}

L. Assländer $(\varangle) \cdot$ G. Hettich · T. Mergner

Neurocenter, Neurological University Clinic, Breisacher Str. 64, 79106 Freiburg, Germany

e-mail: lorenz.asslaender@sport.uni-freiburg.de

L. Assländer · G. Hettich · A. Gollhofer

Institute for Sport and Sportscience, University of Freiburg,

Schwarzwaldstr. 175, 79117 Freiburg, Germany to previous findings that, transferred to a stance control model, suggest a velocity mechanism for tilt compensation and a position mechanism for gravity compensation.

Keywords Humans · Posture control · Multisensory integration $\cdot$ Visual velocity cues $\cdot$ Stroboscopic illumination

\section{Introduction}

Humans combine several sensory cues when controlling bipedal stance during unforeseen external disturbances. With eyes closed, balancing depends mainly on proprioceptive and vestibular cues. Adding vision reduces body sway, an intuitive and long-known phenomenon (see Peterka and Benolken 1995; Horak and Macpherson 1996). The present study addresses this sensory integration issue with focus on the visual cues. The aim of the study is to distinguish the contribution of visual velocity cues from that of visual displacement cues when trying to understand the sensory integration in postural responses to support surface tilt.

Not only visual cues contain velocity information, but also joint angle proprioception and vestibular signals. This is well known, for example, for the muscle spindle contribution to joint angle proprioception. In the vestibular system, angular velocity information from the cupular receptors in the canals can be consciously perceived and, in addition, it appears to be processed centrally with otolith information, allowing humans to distinguish linear motion from tilt (details in Mergner et al. 2009). It has been shown that velocity information plays an important role in posture control (Jeka et al. 2004). Specifically, the posture control model developed by our laboratory (Mergner et al. 2003; Maurer et al. 2006; Mergner 2010) uses vestibular 
and proprioceptive velocity information for estimating and compensating support surface tilt. This mechanism is thought to combine with an estimate of the torque that is evoked by gravitational forces acting on the ankle joint during body lean and is based on vestibular angular displacement information. We hypothesize that both mechanisms profit from adding visual cues and that selectively modifying the visual velocity information specifically affects the estimation and compensation of support surface tilt.

As a means to modify visual velocity information, we resorted to stroboscopic illumination. During stroboscopic illumination, visual motion perception is based on a series of static images of moving visual items presented in rapid succession. The effect is often called apparent motion in vision research (e.g., Wertheimer 1912). It is intuitive from watching films and videos. Upon slowing down the picture presentation, motion perception becomes "partial" or "broken," reminiscent of the jerky movements in old silent films. When such film presentations then become very slow, motion perception passes over into sequences of stationary images with the displayed items in changed position. Although it appears that the correlation-type motion detector of Hassenstein and Reichardt (1956) can provide a parsimonious explanation for apparent motion, it is still difficult to exactly explain the physiological mechanisms (see Introduction to Wertheimer 2012 book under Wertheimer 1912).

However, one may define to some extent stroboscopic effects operationally on the basis of behavioral observations. One effect is a reduction in the optokinetic reflex (OKR), which is known to depend on the velocity of retinal slip. This applies to humans (Schor et al. 1984; Berthoz and Melvill Jones 1985) as well as to monkeys and cats (Flandrin et al. 1990) and occurs in a graded way, depending on the strobe frequency. Stroboscopic illumination also affects the visually evoked self-motion illusion, called circular vection (Schor et al. 1984). An intuitive behavioral consequence of the strobe effect is that the catching of tossed items tends to fail, even if the displacement is well perceived (Croft 1971). The postural control mechanisms that are affected when visual velocity information is degraded by stroboscopic illumination (Amblard et al. 1985; Paulus et al. 1984) are yet to be identified and investigated.

We kept the visual scene in our study stationary. This approach distinguishes it from most studies that investigated the role of visual cues in postural control. These previous studies used visual scene motion, alone or in combination with other stimuli, to separate out the relative contribution of visual, vestibular, and proprioceptive cues when trying to understand the mechanisms of sensory integration in postural control (Bles et al. 1958; Talbott 1980; Peterka and Benolken 1995; Peterka 2002; Jeka et al. 2000; Kuo 2005; Mergner et al. 2005). It is to note, however, that the sway responses to visual scene motion are complicated by cognitive effects (Guerraz et al. 2001; Mergner et al. 2005; Blümle et al. 2006). ${ }^{1}$ A well-known cognitive effect can be observed upon repeated isolated transient scene motions. Presented for the first time to a standing subject, the scene motion tends to evoke a pronounced sway response, while subsequent responses are strongly attenuated, already starting with the second and third presentation (Nashner and Berthoz 1978; Bronstein 1986). This cognitive effect likely also contributes to the saturation of body sway amplitude that is observed when visual scene motion stimuli are presented with increasing magnitude; the saturation starts at a value similar to the detection threshold of vestibular self-motion perception in a condition where ankle proprioceptive feedback is prevented (Peterka and Benolken 1995; Mergner et al. 2005).

The contribution of the cognitive effect to the multisensory integration is still not understood. Underlying the triggering and learning of the cognitive effect appears to be a visual-vestibular conflict, which arises when subjects notice from comparison of visual with vestibular cues that the visual scene is moving and therefore should not be used in perception as a representation of absolute (inertial) space (Zacharias and Young 1981; Mergner and Becker 1990; Mergner et al. 1995). The cognitive effect and the visualvestibular conflict appear to be important in everyday life in that they help to prevent falls and self-motion illusions evoked by visual scene motion. However, for investigating multisensory integration, it is currently still preferable to use the ecologically prevailing situation of a stationary scene, where there is no visual-vestibular conflict, as the cognitive contribution is difficult to estimate. Corresponding studies using a stationary scene and investigating the effects of blurred vision or restricted visual fields found increased spontaneous sway (Paulus et al. 1984), similar to that induced by $3-\mathrm{Hz}$ stroboscopic scene illumination that reduces dynamic visual cues (Amblard et al. 1985). The strobe effect is graded in that spontaneous sway increases with decreasing strobe frequency (Paulus et al. 1984).

Yet, the measure of spontaneous sway in these studies does not allow inferences to be made on how the sensory integration works. The reason is that spontaneous sway is the result of unknown internal noise and its interaction with the control system. In order to allow detailed inferences regarding the sensory integration, it is necessary to apply quantitatively known external disturbances and to compare them with the sway responses they evoke (van der Kooij et al. 2005). This consideration led us to apply support

\footnotetext{
1 Cognitive effects in this context are considered as stemming from conscious or subconscious mental processes that interact with the posture control system based on knowledge or inferences from previous experience, recognizing motion of the visual scene, etc.
} 
surface tilts as external disturbance and to analyze how variations in the visual information content by the stroboscopic illumination affect the tilt responses. In order to experimentally distinguish between velocity and displacement effects in our subjects' tilt responses, we used tilt stimuli that contained a broad range of tilt frequencies, yet with regional differences in the angular displacement and velocity amplitude spectra.

\section{Methods}

Subjects

Seven subjects (28 \pm 3 years of age; 3 females) participated after giving informed consent to participate in this study that was approved by the Ethics Committee of the Freiburg University Clinics. Three subjects were members of the laboratory (two authors), while the other four were naive as to the purpose of the experiments. All subjects were healthy and had no history of neurological disorders. In view of the epileptogenic potential of stroboscopic illumination, particular care was taken to exclude subjects with a history of epilepsy.

Experimental setup, stimuli, and recordings

Subjects stood freely on a custom-made motion platform (Mergner et al. 2003). They were presented with support surface tilts in the sagittal plane with the rotation axis through the ankle joints. Each trial consisted of six successive cycles of a 60.5-s-long pseudorandom ternary sequence (PRTS; frequency range 0.017-2.2 Hz; peakpeak amplitude $4^{\circ}$; Peterka 2002). On the basis of its angular displacement and velocity amplitude spectra (Fig. 1),

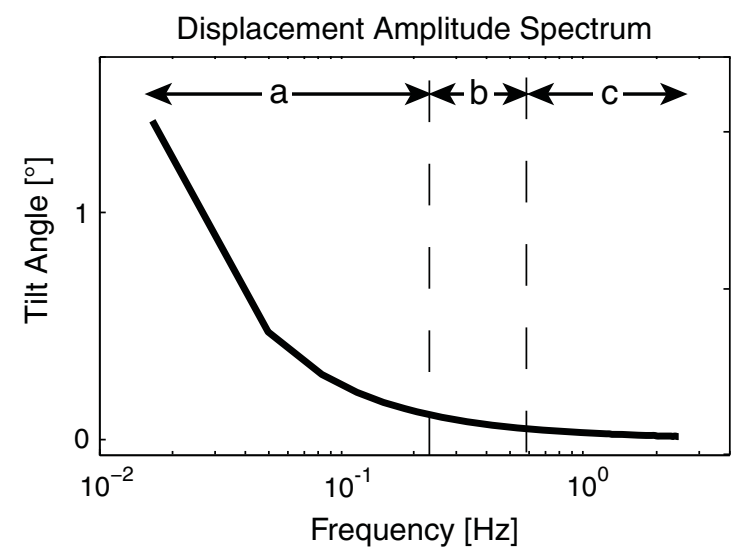

Fig. 1 Angular displacement and velocity amplitude spectra of the PRTS tilt stimulus. The displacement amplitude spectrum (left) is highest in the low frequency range $(a)$ and decreases with increasing three tilt frequency ranges were distinguished (indicated on top of Fig. 1 and Figs. 3, 4): low frequency range of tilts $(0.017-0.182 \mathrm{~Hz})$, where velocity amplitudes were high and constant, while displacement amplitude decreased with increasing tilt frequency from an initially high value; midfrequency range $(0.23-0.44 \mathrm{~Hz})$, where velocity amplitudes were high and displacement amplitudes low; and high frequency range $(0.58-2.20 \mathrm{~Hz})$, where velocity amplitudes decreased with increasing tilt frequency and also displacement amplitudes, already very low, decreased further.

Subjects' leg and trunk excursions were recorded optoelectronically using active markers (Optotrak ${ }^{\circledR}$ 3020; Waterloo, Canada), and body center of mass (COM) angular excursions with respect to the space vertical were calculated from leg and trunk displacements. Tilt stimulus and responses were recorded with an A/D converter at $100 \mathrm{~Hz}$ sampling frequency using a custom-made application programmed in LabView (National Instruments; Austin, USA).

Visual motion cues during stance stemmed from visual information on self-motion, which the moving (here swaying) subject derives with respect to the stationary scene, the laboratory in our experiments. The visual velocity cues then stem from the so-called optic flow (often formalized as velocity vectors of visual images on the retina; Gibson 1950; Koenderink 1986).

For evoking the stroboscopic effect, a number of factors known from vision research were taken into account. This research has shown that smoothness of perceived apparent motion depends on luminance (e.g., photopic or scotopic level), frequency of image presentation (or interstimulus onset interval, ISOI), spatial image shift, and duration of image presentation (Korte 1915). Also, for maintaining a smooth motion perception, changing one parameter requires compensatory changes in the other parameters. In our approach, we aimed at the opposite, at degrading

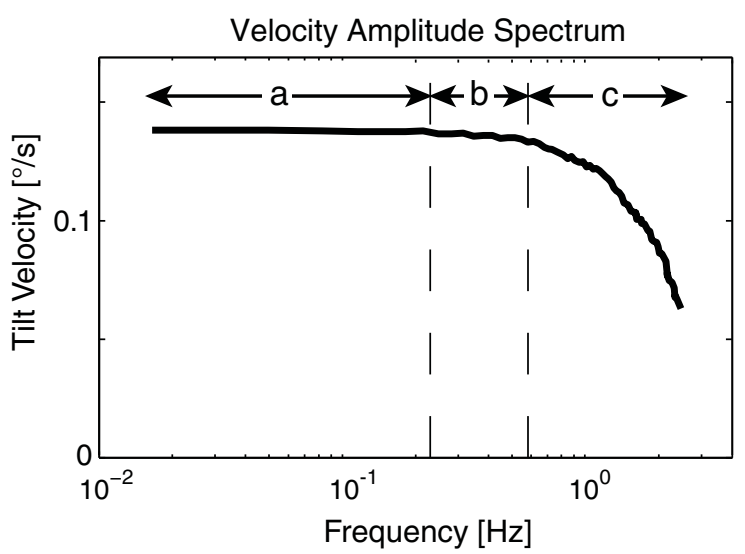

frequency, whereas the velocity amplitude spectrum (right) remains almost constant across the low frequency range and the midfrequency range $(b)$ and decreases in the high frequency range $(c)$ 
motion perception. To do so, we reduced the frequency of image presentation, while maintaining constant luminance and duration of image presentation.

With a conventional stroboscope, however, it proved not possible to maintain a constant mean photopic level of illumination across all strobe frequencies. Therefore, we resorted to a custom-made strobe with super-bright LEDs (Neutral White 4000K; Bridgelux, Livermore, USA). At the chosen mean luminance level (1 lx), where color vision and peripheral vision were maintained, flash duration amounted to $5 \mathrm{~ms}$ and could be kept constant across all stroboscopic conditions. Compared to changes in strobe frequency, this longer than conventional $(0.1 \mathrm{~ms})$ flash duration appears to have little relevance to the strobe effect when judged from apparent motion experiments (Koffka 1931).

In the final experiments, two LEDs were directed to the wall in front of the subjects and one to each side, providing approximately uniform luminance across the visual field. The visual field was unrestricted and comprised approximately half of the laboratory (distance to front wall, $4 \mathrm{~m}$ ) with a rich visual scenario providing a broad range of contrasts and spatial frequencies as well as 3D structures. Seven visual conditions were used: (1) full vision (FuVis) with continuous LED illumination; (2) five conditions with the 5-ms stroboscopic LED illumination at a flash frequency of 4, 8, 16, 32, and $48 \mathrm{~Hz}$ (Strob4, Strob8, Strob16, Strob32, and Strob48, respectively); (3) no vision (NoVis), eyes were closed. After luminance in FuVis was set to 1 1x, mean luminance in the Strob4 to Strob48 conditions was adjusted to that of FuVis by changing the luminous flux of each flash in inverse proportion to the stroboscopic frequency, verifying mean luminance by a luxmeter (Beha ${ }^{\circledR}$; Glottertal, Germany). With flash duration and mean luminance kept constant, subjects' visual acuity and perceived brightness remained constant across the six illumination conditions (see below).

\section{Procedures}

The seven visual conditions were presented in one session as separate trials in randomized order. Between trials, subjects were given breaks of 3-5 min. Prior to each trial, subjects were given time to get familiarized with the visual condition. They were then instructed to "look straight ahead" (or "close the eyes") and "stand upright and comfortable." Listening via earphones to an audio drama minimized auditory orientation cues and distracted subjects' attention from the balancing task. Each subject performed the session twice, either on different days or separated by a break $>45 \mathrm{~min}$.

After each trial (except for NoVis), visual acuity was measured by presenting letters with decreasing size at a fixed viewing distance in a forced-choice procedure (Bach and Kommerell 1998). Visual acuity of all subjects was constant across the illumination conditions (maximum difference $\pm 0.1 \log$ MAR). In addition, subjects were asked to give retrospective reports on: (a) changes in perceived brightness between illumination conditions; in general subjects reported brightness to be constant. (b) Unpleasant feelings with stroboscopic illumination; one subject reported slight nausea in one Strob4 trial, and all subjects considered the stroboscopic trials as slightly unpleasant; more so, the lower the frequency. (c) Subjective stance stability in the stroboscopic trials; all subjects reported this stability to be similar to that in the FuVis condition and clearly better compared to NoVis; this even with Strob4. (d) Subjective motion of the visual scene; the visual scene as a whole was perceived in all visual conditions as stationary in space. During rapid voluntary movements in between trials, small objects were occasionally seen to be transiently moving in a jerky way, while the front wall remained stationary.

\section{Data analysis}

The data recorded in LabView were exported to Matlab (The MathWorks; Natick, USA) for further analyses. The first cycle of the PRTS tilt stimulus was discarded to avoid transient effects. For each of the following five cycles, the power spectra of response (COM angular excursion) and stimulus (support surface tilt) and the corresponding crosspower spectrum were calculated. The frequency response function (FRF) was calculated by dividing the cross-power spectrum by the tilt power spectrum. Gain over frequency was calculated by taking the absolute value of the mean FRF at each frequency, representing the amplitude ratio of the COM response to the tilt stimulus. Zero gain here indicates no stimulus-evoked COM response (COM remaining upright above ankle joints), while a gain of unity indicates that COM response amplitude equals tilt amplitude. Phase, calculated as the inverse tangent of the ratio between the real and imaginary part of the cross-power spectrum, represents the temporal relation between tilt stimulus and COM response. Coherence was calculated by dividing the squared absolute values of the cross-power spectrum by the product of the power spectra of stimulus and response (following the approach of Peterka 2002). Coherence represents a measure for the correlation between PRTS stimulus and response.

Results of test (1st session) and retest (2nd session) of each subject were compared, and tests were repeated upon large differences, before the ten cycles of each subject were treated as one data set. Intersubject variability for gain values was within the range of intrasubject variability, with the exception of one subject who had increased gain values in all conditions. Furthermore, gain values for the low frequency range and the mid-frequency range of the tilts showed an essentially normal distribution across tests in 
each given illumination condition. Therefore, parametric statistics were applied, whose results are in line with those based on nonparametric statistics that were calculated for comparison in view of the limited number of subjects.

All mean values and standard deviations were calculated in the complex domain before expressing them as gain and phase. The mean of the ten cycles of each subject was calculated, and the overall mean, standard deviation (giving intersubject variability), and significance tests (Student's $t$ test for repeated measures) were calculated thereof.

\section{Results}

Figure 2 shows the support surface tilt stimulus sequence in the time domain and the COM responses evoked in the
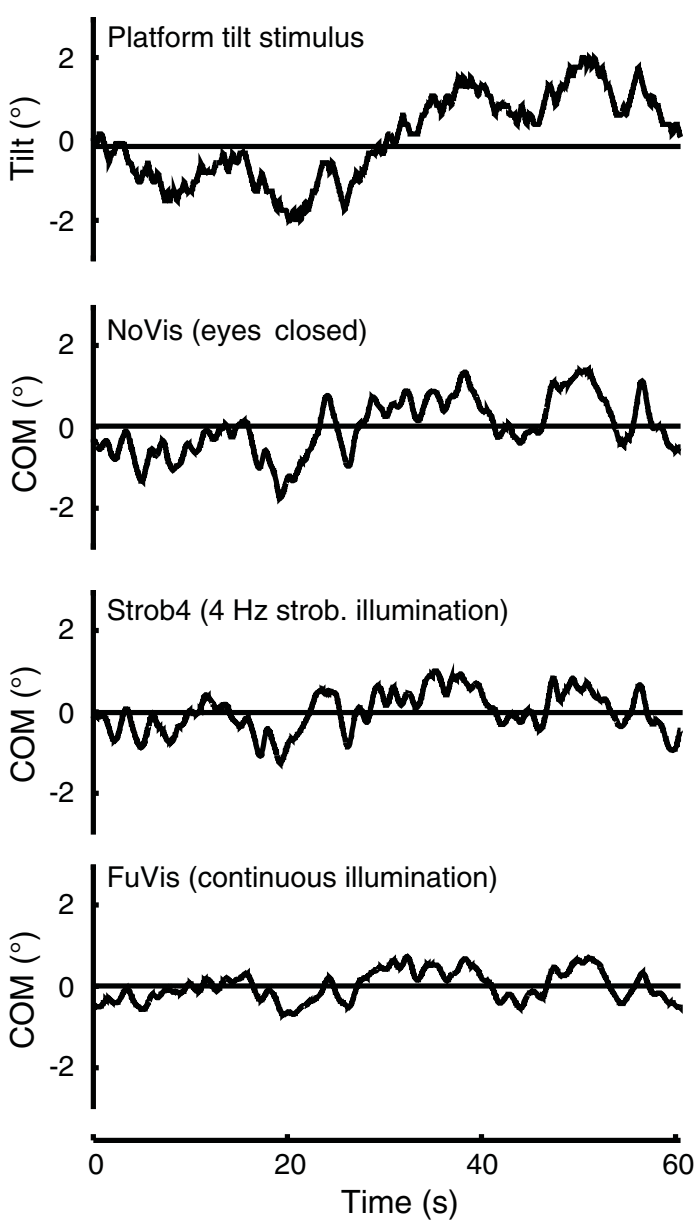

Fig. 2 Time series of platform tilt stimulus and COM sway responses of a representative subject for the three example conditions NoVis (eyes closed), Strob4 (4-Hz stroboscopic illumination), and FuVis (eyes open, continuous illumination). The stimulus consists of a 60.5-s pseudorandom ternary sequence (PRTS) of tilts with peakpeak amplitude of $4^{\circ}$. COM responses represent means of five stimulus repetitions
NoVis, Strob4, and FuVis conditions from one representative subject. In the NoVis condition (eyes closed), the COM excursions resemble the platform excursions, demonstrating incomplete compensation of the support surface tilt stimulus. Compensation for the stimulus here means producing active ankle torque that counteracts COM rotation evoked by muscular-skeletal ankle stiffness and gravitational ankle torque. In FuVis (continuous illumination), the COM excursions are clearly smaller, while in the Strob4 condition they fall in between those for the NoVis and FuVis conditions.

Figure 3 shows mean gain, phase, and coherence curves plotted over tilt frequency. The upper and lower borders of the gain data are given by the gain curves for the NoVis and FuVis conditions, respectively. The NoVis gain curve starts at $0.017 \mathrm{~Hz}$ with a gain value of $G=0.58$ and increases with increasing tilt frequency, reaching a plateau at about $0.2 \mathrm{~Hz}(G=1.4)$. Above $0.5 \mathrm{~Hz}$, the gain decreases, reaching $G=0.2$ at $2.2 \mathrm{~Hz}$. The corresponding FuVis curve shows similar gain-over-tilt frequency characteristics, but the gain values are almost half. Despite this gain difference, the mean phase curves of NoVis and FuVis resemble each other. Responses in both conditions are essentially in phase with the tilt at $0.2 \mathrm{~Hz}$, showing a slight phase lead at lower frequencies $\left(\approx 30^{\circ}\right.$ at $\left.0.017 \mathrm{~Hz}\right)$, and develop a phase lag above $0.2 \mathrm{~Hz}$, reaching $\approx 200^{\circ}$ at $2.2 \mathrm{~Hz}$. There were only minor phase differences between the two visual conditions. At low and high tilt frequencies, the phase in the NoVis condition lagged that for FuVis by $\approx 10^{\circ}$ ( $p=0.06$ at $0.017 \mathrm{~Hz}$ tilt frequency and $p=0.14$ at $2.2 \mathrm{~Hz}$ ). The coherence curves for both conditions fell in between 0.5 and 1 . This applied from $0.017 \mathrm{~Hz}$ to approximately $1.0 \mathrm{~Hz}$ tilt frequency, after which it showed a pronounced decrease below 0.5 .

In the stroboscopic conditions, the gain and coherence curves fell in between the curves for FuVis and NoVis, with few exceptions only. The phase curves essentially coincided with those for FuVis and NoVis. Figure 3 includes the gain, phase, and coherence curves of the two stroboscopic conditions, Strob4 and Strob16. The gain curve for Strob16 is close to that of FuVis at the lowest and the highest tilt frequencies, but deviates from it at most of the intermediate frequencies. The gain curve for Strob4 is slightly higher than the Strob16 gain curve at low and high tilt frequencies, showing the largest deviation in the mid-frequency range, where it approaches the NoVis gain curve. Thus, increasing stroboscopic frequency from 4 to $16 \mathrm{~Hz}$ yields a pronounced effect in the mid-frequency range of tilts and clearly smaller effects in the low frequency range and the high frequency range.

In a quantitative comparison of gain across the seven visual conditions, we averaged the gain data separately for each of the three ranges of tilt frequency that we 
Fig. 3 Visual effects on COM tilt responses. Plotted are mean gain, phase, and coherence curves ( $n=7$ subjects, 10 tilt cycles each) as a function of tilt frequency for the conditions NoVis, FuVis, Strob4, and Strob16. Gain indicates the ratio of the response amplitude to the stimulus amplitude (Gain $=0$, no COM excursion in space in response to the tilt; Gain $=1$, COM response amplitude equals tilt amplitude). The gain curves of the two stroboscopic conditions Strob4 and Strob16 tend to fall in between those for NoVis and FuVis
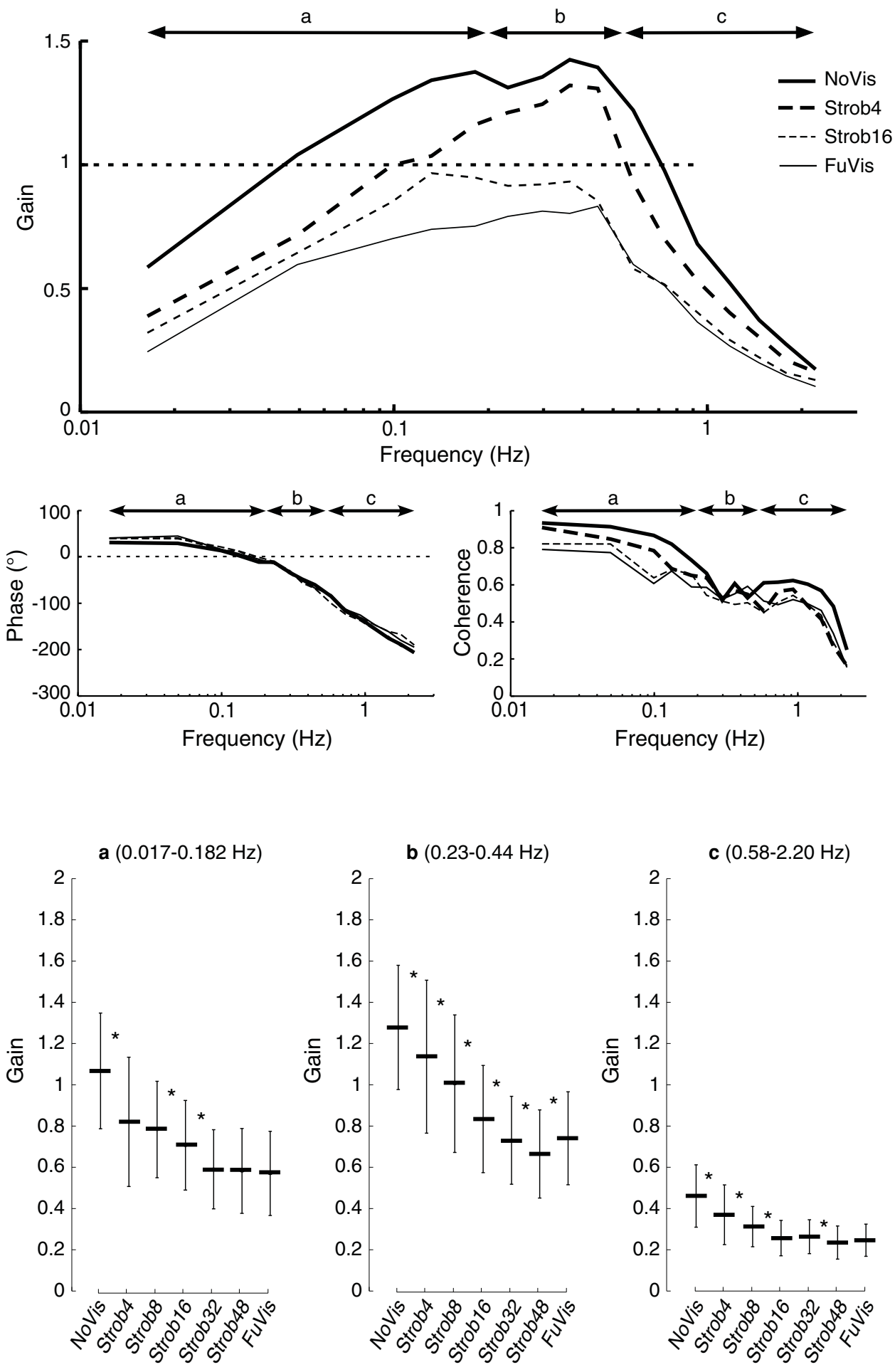

Low frequency range (averaged tilt velocity amplitude high, displacement amplitude intermediate). Gain in Fig. 4a is reduced by a large step from NoVis to Strob4 $(p<0.001)$, representing clearly better tilt compensation in the lowest strobe frequency condition as compared distinguished on the basis of the angular displacement amplitude and velocity amplitude spectra of the stimulus (Fig. 1). The averaged mean gain values and their intersubject variability in terms of standard deviation are shown in Fig. 4:
Fig. 4 Mean gain values and intersubject variability of $\mathrm{COM}$ for the low frequency range (a) the mid-frequency range (b), and the low frequency range (c) seven visual conditions (abscissas). Statistically significant differences between neighboring conditions $(p<0.05)$ are indicated by asterisks 
to NoVis. Smaller steps followed with increasing strobe frequency from Strob8 via Strobl6 to Strob32, where approximately the values for Strob48 and FuVis were reached.

Mid-frequency range (averaged velocity amplitude high, displacement amplitude low). Similar as in the low frequency range, gain is reduced in steps from NoVis via Strob4, Strob8, Strob16, Strob32 to Strob48, but the step from NoVis to Strob4 is clearly smaller and the succeeding steps more regular and relatively larger compared to the low frequency range. All steps are statistically significant. Gain for Strob32 is again similar to that for FuVis, while that for Strob48 is slightly lower.

High frequency range (averaged velocity amplitude intermediate, displacement amplitude very low). Similar as before, yet on a clearly lower averaged gain level, gain is reduced with increasing strobe frequency, but the effect appears to level off at Strobl6.

Noticeably, despite these changes in gain, subjects experienced their stance stability in all stroboscopic trials to be similar to that in the FuVis condition and clearly better compared to NoVis (see "Methods," retrospective reports).

\section{Discussion}

Focusing on the visual contribution to posture control, this study explores the distinction between visual displacement and velocity cues, to better understand the way in which visual information renders its effects when interacting with vestibular and proprioceptive cues. By keeping the visual scene stationary, we tried to avoid the cognitive effect that tends to arise with moving visual scenes from visual-vestibular conflict (see "Introduction"). Using a systems analysis approach, we studied how the graded reduction in visual velocity cues through stroboscopic illumination affects the postural responses evoked by an exactly known external stimulus, the support surface tilt. Tilts were presented in a pseudorandom sequence with defined angular displacement and velocity amplitude spectra across a broad range of tilt frequencies. A first finding was that the visual input clearly affects the gain and hardly the phase of the tilt response, which is in line with previous work (Peterka and Benolken 1995; Maurer et al. 2000, 2006; Peterka 2002). This finding allows us to focus in the following on the gain curves.

Two effects can be separated in the experimental findings. One is a graded reduction in the sway response gains with increasing strobe frequency, which is visible throughout the measured frequency range, but shows the largest effect in the mid-frequency range. The other is a relatively large gain drop from NoVis to Strob4, which occurred mainly in the low frequency range. This finding is in accordance with the results during quiet stance (Paulus et al. 1984; Amblard et al. 1985), but provides further information on how the stroboscopic illumination affects the postural control system when this is compensating for support surface tilts.

Before addressing the interpretation of our results, we briefly explain the two disturbance estimation and compensation mechanisms, which we previously suggested for the eyes closed condition on the basis of human stance control findings (Mergner et al. 2003; Mergner et al. 2005; Mergner 2010):

1. Mechanism 1: A gravitational ankle torque estimation and compensation mechanism. It estimates the body angular displacement with respect to the gravitational vertical, using vestibular information. Parameter identification suggested that the estimate contains an angular displacement detection threshold. The task of Mechanism 1 is to compensate body lean-related gravitational ankle torque, contributing to the compensation for platform tilts primarily at lower frequencies (compare Fig. 7a in Maurer et al. 2006, who used sinusoidal tilts at $0.025-0.4 \mathrm{~Hz}$ ).

2. Mechanism 2: A support surface tilt estimation and compensation mechanism. It estimates support surface tilt, involving vestibular and proprioceptive angular velocity cues. Parameter identification suggested an angular velocity detection threshold. Mechanism 2 is used to provide the ankle torque that is required to compensate for the tilt-related rotation of the feet in space. It contributes to the compensation for the tilt mainly at higher frequencies (compare again Fig. 7a in Maurer et al. 2006).

We consider it as plausible that the visual displacement information in the eyes open condition of the present study contributes mainly to Mechanism 1 and the visual velocity information mainly to Mechanism 2. The current experimental results are qualitatively consistent with this assumption. Specifically, if the stroboscopic illumination primarily reduces the visual velocity information, while preserving visual position information, the stroboscopic effects from FuVis to Strob4 should influence the gain mainly at higher tilt frequencies, here corresponding to the mid-frequency range and slightly beyond (up to about $0.6 \mathrm{~Hz}$; compare Fig. 3). Furthermore, it can be assumed that the visual velocity information in the Strob4 condition is strongly reduced and the gain difference observed between the Strob4 and the NoVis conditions is caused mainly by visual angular displacement information. Given that the visual angular displacement information contributes to Mechanism 1 , one would expect to find a large influence on the 
Strob4 to NoVis gain difference mainly at lower frequencies. In fact, the largest Strob4-NoVis gain difference was found in the low frequency range (Figs. 3, 4).

The experimental findings are thus consistent with our hypothesis that visual angular displacement cues are combined with vestibular angular displacement cues in Mechanism 1 of the human stance control mechanism and that visual angular velocity cues are combined with vestibular and proprioceptive angular velocity cues in Mechanism 2. In order to go one step further and to achieve quantitative predictions, modeling work is needed to generate a new model that combines the present findings with our previous model. The new model may then suggest additional experiments that can further test our sensory integration concept of how humans compensate for support surface tilt.

Acknowledgments We would like to thank Frank Hüthe for building the strobe device and for providing technical support and Jörg Dietterle for helping in performing the experiments.

\section{References}

Amblard B, Crémieux J, Marchand AR, Carblanc A (1985) Lateral orientation and stabilization of human stance: static versus dynamic visual cues. Exp Brain Res 61:21-37

Bach M, Kommerell G (1998) Determining visual acuity using European normal values: scientific principles and possibilities for automatic measurement. Klinische Monatsblätter für Augenheilkunde 212:190-195

Berthoz A, Melvill Jones G (1985) Adaptive mechanisms in gaze control. Elsevier Science Publishers B.V. (Biomedical Division), Amsterdam, pp 106-108

Bles W, Kapteyn TS, Brandt T, Arnold F (1958) The mechanism of physiological height vertigo. II. Posturography. Acta Otolaryngol 89:534-540

Blümle A, Maurer C, Schweigart G, Mergner T (2006) A cognitive intersensory interaction mechanism in human postural control. Exp Brain Res 173:357-363

Bronstein AM (1986) Suppression of visually evoked postural responses. Exp Brain Res 63:655-658

Croft T (1971) Failure of visual estimation of motion under strobe. Nat 5302:397

Flandrin JM, Courjon JH, Magnin M, Arzi M (1990) Horizontal optokinetic responses under stroboscopic illumination in cat, monkey and man. Exp Brain Res 81:59-69

Gibson JJ (1950) The perception of the visual world. Houghton Mifflin Company, Boston

Guerraz M, Thilo KV, Bronstein M, Gresty M (2001) Influence of action and expectation on visual control of posture. Brain Res Cogn Brain Res 11:259-266

Hassenstein B, Reichardt W (1956) Systemtheoretische Analyse der Zeit-, Reihenfolgen- und Vorzeichenauswertungen bei der Bewegungsperzeption des Rüsselkäfers Chlorophanus. Zeitschrift für Naturforschung 11b:513-524 (Systems theory analysis of time, sequence, and sign evaluation in the motion perception of the proboscis beetle Chlorophanus)

Horak F, Macpherson J (1996) Postural orientation and equilibrium. In: Rowell L, Shepherd J (eds) Handbook of physiology 1.
Exercise: regulation and integration of multiple systems. Oxford University Press, New York, pp 255-292

Jeka JJ, Oie KS, Kiemel T (2000) Multisensory information for human postural control: integrating touch and vision. Exp Brain Res 134:107-125

Jeka JJ, Kiemel T, Creath R, Horak F, Peterka RJ (2004) Controlling human upright posture: velocity information is more accurate than position or acceleration. J Neurophysiol 92:2368-2379

Koenderink JJ (1986) Optic flow. Vis Res 26:161-179

Koffka K (1931) Die Wahrnehmung von Bewegung. In: Bethe A (ed) Handbuch der normalen und pathologischen Physiologie, vol XII/2. Springer, Berlin, pp 1166-1214

Korte A (1915) Kinematoskopische Untersuchungen. Z Psychol 72:193-206

Kuo AD (2005) An optimal state estimation model of sensory integration in human postural balance. J Neur Eng 2:235-249

Maurer C, Mergner T, Bolha B, Hlavacka F (2000) Vestibular, visual, and somatosensory contributions to human control of upright stance. Neursci Lett 281:99-102

Maurer C, Mergner T, Peterka RJ (2006) Multisensory control of human upright stance. Exp Brain Res 171:231-250

Mergner T (2010) A neurological view on reactive human stance control. Ann Rev Control 34:177-198

Mergner T, Becker W (1990) Perception of horizontal self-rotation: multisensory and cognitive aspects. In: Warren R, Wertheim AH (eds) Perception and control of self-motion. Lawrence Erlbaum, Hillsdale London, pp 219-263

Mergner T, Schweigart G, Kolev O, Hlavacka F, Becker W (1995) Visual-vestibular interaction for human ego-motion perception. In: Mergner T, Hlavacka F (eds) Multisensory control of posture. Plenum Press, New York, pp 157-168

Mergner T, Maurer C, Peterka RJ (2003) A multisensory posture control model of human upright stance. Prog Brain Res 142:189-201

Mergner T, Schweigart G, Maurer C, Blümle A (2005) Human postural responses to motion of real and virtual visual environments under different support base conditions. Exp Brain Res 167:535-556

Mergner T, Schweigart G, Fennel L (2009) Vestibular humanoid postural control. J Physiology Paris 103:178-194

Nashner L, Berthoz A (1978) Visual contribution to rapid motor responses during postural control. Brain Res 150:403-407

Paulus WM, Straube A, Brandt T (1984) Visual stabilization of posture. Physiological stimulus characteristics and clinical aspects. Brain 107:1143-1163

Peterka RJ (2002) Sensorimotor integration in human postural control. J Neurophysiol 88:1097-1118

Peterka RJ, Benolken MS (1995) Role of somatosensory and vestibular cues in attenuating visually induced human postural sway. Exp Brain Res 105:101-110

Schor CM, Lakshminarayanan V, Narayan V (1984) Optokinetic and vection responses to apparent motion in man. Vis Res 24:1181-1187

Talbott RE (1980) Postural reactions of dogs to sinusoidal motion in the peripheral visual field. Am J Physiol 239:R71-R79

van der Kooij H, van Asseldonk E, van der Helm FCT (2005) Comparison of different methods to identify and quantify balance control. J Neurosci Methods 145:175-203

Wertheimer M (1912) Ueber das Sehen von Scheinbewegungen und Scheinkoerpern. Z Psychol 61:161-265 (English translation in: Wertheimer M (2012). On Perceived Motion and Figural Organization. Spillmann L (ed), MIT Press, Cambridge)

Zacharias G, Young L (1981) Influence of combined visual and vestibular cues on human perception and control of horizontal rotation. Exp Brain Res 41:159-171 